\section{La percezione della web atmosphere tra i visitatori del centro commerciale}

\author{
Marco Cioppi - Elisabetta Savelli - Federica Tombari
}

\begin{abstract}
Obiettivo del paper: Il paper analizza le variabili della websiteatmosphere utilizzabili nella progettazione del sito web di un centro commerciale ed approfondisce l'importanza dei diversi attributi assumendo la prospettiva del potenziale utente ed $i$ suoi atteggiamenti nei confronti del format.

Metodologia: Il lavoro si basa su un'indagine empirica, condotta tramite questionario, rivolta ad un campione di 438 studenti universitari, di età compresa tra i 18 e 30 anni.

Risultati: L'indagine rivela l'esistenza di atteggiamenti diversi degli utenti nei confronti della web atmosphere a seconda del comportamento di shopping prevalente ed unattenzione diffusa verso gli attributi social del sito web che favoriscono l'interattività.

Limiti della ricerca: Il campione indagato è composto da soli studenti universitari con uno stile di vita particolare. In futuro, si ritiene necessario estendere l'indagine ad una popolazione piu ampia e prendere in considerazione anche la prospettiva dell'impresa commerciale.

Implicazioni pratiche: Investimenti online mirati a rafforzare la presenza e le promozioni sui social network ed altri strumenti di comunicazione interattiva (forum, chat...) potrebbero costituire efficaci leve per incrementare lattrattività del sito web e rafforzare i processi di fidelizzazione.
\end{abstract}

Originalità del paper: In letteratura non si riscontrano modelli di websiteatmosphere specificatamente riferiti agli shopping center (e più in generale al contesto retail). Inoltre, il contributo focalizza l'attenzione sulla dimensione social della web atmosphere, anchessa meno esplorata in letteratura.

Parole chiave: web atmosphere; web marketing; marketing esperienziale; centro commerciale

Purpose of the paper: The paper focuses on the analysis of atmospheric cues of shopping center' websites. The aim of the study is to understand the importance of different atmospherics, by considering the customer perspective and his/her attitude towards a shopping center.

Methodology: The research is based on a survey carried out on a sample of 438 college students, aged between 18 and 30 years.

Findings: Findings reveal that customers with different shopping center's attitudes give different importance to the atmospheric cues. Moreover respondents usually give a lot of importance to websiteattributes that promote social interactivity.

Research limits: The sample includes only students having similar lifestyle. It seems necessary to extend the investigation to a wider population and to take into 
sinergie Vol. 34, N. 100, 2016

account also the retailer's perspective in order to better analyze the management of online atmosphere.

Practical implications: Online investment aimed at strengthening the presence and promotions on social networks and other interactive applications (forum, chat ...) could be helpful to increase the websiteattractiveness and to improve the customers retention.

Originality: Literature on websiteatmosphere applied to the shopping centers (and in general to the retail sector) is very limited. Moreover, this contribution focuses on the social dimension of the websiteatmosphere, that was little explored in past studies.

Key words: web atmosphere; web marketing; experiential marketing; shopping center

\section{Introduzione $e^{1}$}

Il sito web aziendale è uno strumento di comunicazione molto importante per raggiungere il consumatore e sempre più spesso diviene un canale di vendita alternativo o parallelo alla distribuzione tradizionale (Barlow et al., 2004; Eurostat, 2014). Ne consegue la necessità di progettare siti web efficaci ed attraenti sia sotto il profilo comunicativo/relazionale che commerciale.

La letteratura internazionale è ricca di contributi che affrontano il tema della "qualità" di un sito web, comunemente identificata con dei costrutti multidimensionali, la cui definizione varia a seconda delle categorie di prodotto e dei settori presi in considerazione (Aladwani e Plavia, 2002).

In questo lavoro, il concetto di websitequality viene riferito alla Web Atmosphere (WA), intesa come l'insieme degli elementi del sito web la cui progettazione può determinare effetti sugli utenti, sia in termini emozionali che comportamentali (Dailey, 2004). Il contesto di analisi è quello distributivo, riguardando, in particolare, il format dei centri commerciali.

La ricerca ha per oggetto lo studio delle variabili della WA (c.d. atmospherics) utilizzabili nella progettazione del sito web di un centro commerciale e vuole approfondire quali attributi sono più rilevanti assumendo la prospettiva del potenziale utente. Il quesito che ci si pone, in particolare, è il seguente: in che modo gli atteggiamenti degli utenti nei confronti del centro commerciale possono condizionare le finalità per cui il sito web viene utilizzato e, di conseguenza, gli elementi di quest'ultimo dai quali si è maggiormente attratti?

Le motivazioni dello studio sono diverse.

Sebbene il tema del web design applicato al contesto retail abbia ricevuto crescente attenzione in letteratura (Kim e Stoel, 2004; Nikolaeva, 2006; Wang et al., 2007; Dawson e Kim, 2010; Ainsworth e Ballantine, 2014; Molla-Descals et al., 2014), gli studi focalizzati sui centri commerciali sono limitati. Nondimeno, il sito web presenta grandi potenzialità per tali

I risultati preliminari di questa ricerca sono stati presentati in occasione dell'XI Convegno Annuale della Società Italiana Marketing (Cfr. Cioppi e Savelli, 2014). 
strutture, sia in termini di obiettivi che di contenuti comunicazionali. Oltre alla funzione informativa, il sito potrebbe svolgere un importante ruolo di attrazione al punto vendita fisico. Favorendo la condivisione dei contenuti e l'interattività il sito comunica, infatti, meglio di altri strumenti, il carattere emozionale ed intrattenitivo su cui si basa la distintività del format (Banerjee, 2012). La sua navigazione potrebbe, pertanto, incrementare il desiderio e la curiosità degli utenti di visitare la struttura fisica. In aggiunta, poiché Internet possiede, di per sé, una forte connotazione intrattenitiva, una progettazione adeguata del sito web potrebbe essere complementare rispetto allofferta complessiva di entertainment del centro commerciale (Kim, 2002), contribuendo ad arricchire la sua funzione sociale e relazionale. La criticità del sito web per i centri commerciali emerge anche considerando alcune ricerche che hanno approfondito il fenomeno dello sviluppo degli shopping center online (Shim e Suh, 2010; Kwon et al., 2014). Ahn, Seewon e Ingoo (2004) rilevano l'importanza, anche per le strutture tradizionali, di gestire efficacemente il sito web in modo da affrontare al meglio la competizione dei centri virtuali, il cui sito tende ad essere molto curato e ben strutturato. Shim e Suh (2010) suggeriscono, invece, l'importanza di un'adeguata gestione del sito web per supportare le attività di analisi della domanda e di gestione delle relazioni.

Nonostante queste potenzialità, il sito web di molti centri commerciali è statico e semplice, assolvendo un ruolo di mera vetrina informativa, attraverso cui si comunica il proprio posizionamento, i giorni e gli orari di apertura ed i negozi presenti all'interno della struttura (CNCC, 2015).

Nasce da qui l'interesse di questa ricerca e la volontà di studiare come può essere gestito il sito web di un centro commerciale per sfruttare al meglio le sue potenzialità comunicative e relazionali, oltre che informative.

La scelta di focalizzarsi sul concetto di WA, in particolare, si lega alla considerazione che tale concetto è espressivo della componente esperienziale ed emotiva dellofferta degli shopping center. La dimensione emozionale ed edonistica dello shopping rappresenta, infatti, il principale elemento di differenziazione del centro commerciale (De Nisco e Napolitano, 2006; Gallucci e Poponessi, 2008) e il concetto di WA esprime al meglio tale connotazione, collegandosi direttamente alla shopping experience (Eroglu et al., 2003).

Il lavoro si sviluppa attraverso un'analisi della letteratura focalizzata sui temi della comunicazione online e della WA ed un'indagine empirica, di tipo quantitativo, condotta su un campione di potenziali utenti.

I risultati della ricerca rivelano che i giovani apprezzano la possibilità offerta dal sito web di metterli in contatto con il centro commerciale o altri utenti ad esso interessati e dichiarano di essere tendenzialmente molto attratti dagli elementi del sito web di natura social, che favoriscono l'interattività $\mathrm{e}$ la condivisione dei contenuti. Da ciò derivano implicazioni interessanti in merito alla gestione degli investimenti online mirati ad incrementare le visite e rafforzare i processi di fidelizzazione. 


\section{sinergie}

Vol. 34, N. 100, 2016

\section{Background teorico e framework analitico di riferimento}

A partire dagli anni '90 sono stati prodotti numerosi studi finalizzati a comprendere le dimensioni-chiave su cui puntare per progettare siti web efficaci, in grado di attrarre e fidelizzare i consumatori (Nour e Fadlalla, 2000; Chen e Yen, 2004; Tan e Wei, 2006; Hernandez et al., 2009; Belanche et al., 2012). Un indirizzo particolare si è sviluppato, dagli anni 2000, attorno al tema della WA. Questo concetto, comprende molte variabili dell'ambiente virtuale considerate negli studi sulla website quality. La sua peculiarità sta nel richiamo forte e diretto al concetto di shopping experience (Eroglu et al., 2003). Essa deriva, infatti, dalla nozione tradizionale di atmosfera elaborata con riferimento al contesto fisico, la quale prende spunto dal filone del consumo edonistico (Hirschman e Holbrook, 1982) e rappresenta l'insieme di stimoli, tangibili e non, che possono essere gestiti per creare ambienti fisici piacevoli ed attraenti, in grado di sedurre il consumatore e favorire la soddisfazione dei suoi bisogni immateriali ed edonistici (Turley e Milliman, 2000).

Nonostante il crescente interesse verso lo studio della WA, la ricerca empirica su questo tema appare ancora piuttosto limitata (Gatautis e Vaiciukynaite, 2013). Alcuni autori hanno approfondito singoli atmospherics, quali la navigabilità (Taylor e England, 2006), la musica (Kim e Lennon, 2012) e i colori (Koo e Ju, 2010). Uno studio recente di Manganari et al., (2009) ha portato, invece, alla definizione di una tassonomia concettuale secondo cui la WA è composta da quattro componenti fondamentali: (1) virtual layout and design (VLAY); (2) virtual atmospherics (VATM); (3) virtual theatrics (VTHE) e (4) virtual social presence (VSOC). Tale tassonomia è stata successivamente completata da Gatautis e Vaiciukynaite (2013) con l'aggiunta di ulteriori variabili (Tab. 1).

Il modello che ne deriva è esaustivo in quanto comprende nella definizione di WA sia la dimensione funzionale che informativa e comunicazionale del sito web. Esso, tuttavia, è molto generico e non considera le peculiarità dei contesti aziendali.

Con riferimento al settore retail, e soprattutto ai centri commerciali, non sono stati trovati modelli di analisi ad hoc. Nello stesso tempo, come si è detto, questo contesto presenta caratteristiche tali da rendere potenzialmente interessante lo studio di un modello specifico di atmospherics su cui basare la progettazione del sito web, attraverso un'esplicitazione delle variabili quanto più precisa possibile.

Integrando il modello di Gatautis e Vaiciukynaite (2013) con precedenti ricerche sul websitedesign e la WA, si propone in questo lavoro un framework di attributi in grado di rispecchiare le specificità del contesto. L'elenco di variabili, raggruppate nelle quattro dimensioni definite da Gatautis and Vaiciukynaite è sintetizzato in tabella 1 . 
Tab. 1: Le variabili della web atmosphere applicate ai centri commerciali

\begin{tabular}{|c|c|c|c|}
\hline \multirow[t]{2}{*}{$\begin{array}{c}\text { Modello concettuale di } \\
\text { Gatautis e Vaiciukynaite } \\
(2013)\end{array}$} & \multicolumn{2}{|r|}{ Attributi } & \multirow[t]{2}{*}{ Riferimenti } \\
\hline & & Descrizione & \\
\hline \multicolumn{4}{|c|}{ VLAY1 } \\
\hline $\begin{array}{l}\text { Grid layout } \\
\text { Free-form layout } \\
\text { Racetrack layout } \\
\text { Navigational design } \\
\text { - tree hierarchical } \\
\text { structure, site map } \\
\text { index, search engine; } \\
\text { - web it e co n t e n t } \\
\text { ranking and } \\
\text { grouping; } \\
\text { menu, links }\end{array}$ & 5 & $\begin{array}{l}\text { Facilità di navigazione del } \\
\text { sito (presenza di un percorso } \\
\text { guidato per la navigazione delle } \\
\text { diverse pagine) } \\
\text { Funzionalità di ricerca avanzate } \\
\text { (mappa del sito, motore } \\
\text { di ricerca interno, menu } \\
\text { permanente del sito) } \\
\text { Presenza di link che rimandano } \\
\text { ad altri siti web collegati (es.: } \\
\text { link che rimandano a siti web } \\
\text { delle insegne presenti nel centro } \\
\text { commerciale) } \\
\text { Accessibilità del sito web (= } \\
\text { buona indicizzazione } \\
\text { principali motori di ricerca) } \\
\text { Velocità di navigazione }\end{array}$ & $\begin{array}{l}\text { Baloglu e Peckan, 2006; Dreze e } \\
\text { Zufryden, 2004; Hernández et al., } \\
\text { 2009; Miranda e Benagil, } 2004 \\
\text { Belanche et al., 2012; Cao et al., } \\
\text { 2005; Hernández et al., 2009; } \\
\text { Kim e Lin, 2001; Nielsen, 2000; } \\
\text { Schneiderman, 1998; Weinberg, } \\
2000 \\
\text { Baiet al., 2008; Baloglu e Peckan, } \\
2006\end{array}$ \\
\hline & & VATM2 & \\
\hline $\begin{array}{l}\text { Background colour } \\
\text { Colour scheme } \\
\text { \% of white space } \\
\text { Background music } \\
\text { Scent appeal } \\
\text { Touch appeal } \\
\text { Information content } \\
\text { and structure } \\
\text { Aesthetic design } \\
\text { W e b s i t e b r a n d } \\
\text { personality }\end{array}$ & 8 & 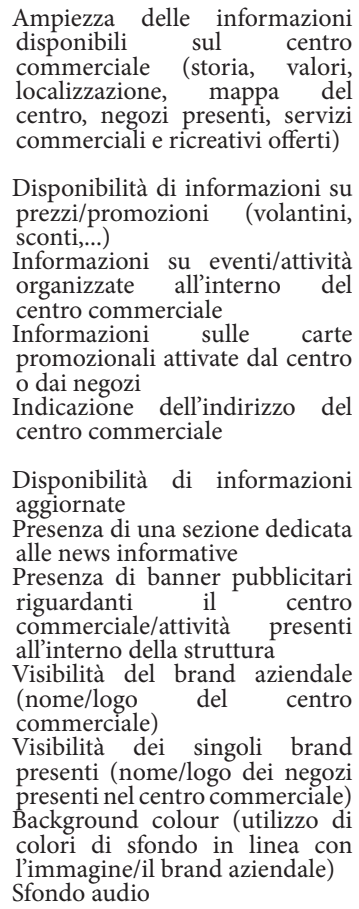 & $\begin{array}{l}\text { Baloglu e Peckan, 2006; Chen } \\
\text { e Yen, 2004; Hernández et al., } \\
\text { 2009; Wan, 2000 } \\
\text { DeLone e McLean, } 1992 \\
\text { Baloglu e Peckan, } 2006 \\
\text { Baloglu e Peckan, } 2006\end{array}$ \\
\hline
\end{tabular}


sinergie

Vol. 34, N. 100, 2016

\begin{tabular}{|c|c|c|c|}
\hline \multicolumn{4}{|c|}{ VTHE $^{3}$} \\
\hline $\begin{array}{l}\text { Animation techniques } \\
\text { Image interactivity } \\
\text { Vividness } \\
\text { interactivity }\end{array}$ & & $\begin{array}{l}\text { Flash animation } \\
\text { Photogallery } \\
\text { riguardante il centro } \\
\text { commerciale } \\
\text { Photogallery riguardante i } \\
\text { singoli negozi } \\
\text { Videogallery } \\
\text { riguardante il centro } \\
\text { commerciale } \\
\text { Videogallery riguardante i } \\
\text { singoli negozi } \\
\text { Tour virtuale del centro } \\
\text { commerciale }\end{array}$ & Baloglu e Peckan, 2006 \\
\hline \multicolumn{4}{|c|}{ VSOC $^{4}$} \\
\hline $\begin{array}{l}\text { Web counter } \\
\text { Comment from other } \\
\text { visitors } \\
\text { Crowding } \\
\text { Avatars } \\
\text { Virtual communities } \\
\text { Recommendations } \\
\text { Communication }\end{array}$ & & $\begin{array}{l}\text { Presenza di un web-counter } \\
\text { Presenza sezione "lascia un } \\
\text { commento" } \\
\text { Visualizzazione del } \\
\text { numero di persone } \\
\text { contemporaneamente sul sito } \\
\text { Presenza di forum di } \\
\text { discussione } \\
\text { Links ai principali social } \\
\text { network }\end{array}$ & $\begin{array}{l}\text { Chenet al., 2010; Kim e Stoel, } \\
\text { 2004; Ranganathan e Ganapathy, } \\
2002 \\
\text { Kim e Stoel, } 2004 \\
\text { Jiang et al., } 2010 \\
\text { Bai et al., } 2008 \\
\text { Bai et al., } 2008 \\
\text { Bai et al., } 2008 \\
\text { Bai et al., } 2008\end{array}$ \\
\hline
\end{tabular}

Riguarda principalmente la navigabilità/accessibilità/usabilità del sito.

Riguarda principalmente il contenuto informativo del sito (varietà e dettaglio) e il modo in cui le informazioni sono strutturate e presentate (grafica, colori, ecc.).

3 Riguarda principalmente la dimensione estetica del sito (come sono presentati testi e immagini).

4 Riguarda principalmente la capacità del sito di far interagire l'impresa con gli utenti e di stimolare le relazioni tra questi.

Fonte: ns. elaborazione

Molte di queste variabili sono state approfondite in precedenti ricerche. L'impatto positivo sul comportamento dell'utente è stato spesso dimostrato, soprattutto con riferimento alla dimensione fisica dellatmosfera online (Manganari et al., 2009).

Meno esplorati appaiono, invece, gli effetti sul consumatore prodotti dalla dimensione sociale della WA, attinenti gli aspetti comunicazionali ed interattivi del sito (Loiacono et al., 2007; Hausman e Siekpe, 2009), sebbene 
la letteratura tradizionale (Babin e Attaway, 2000; Baker et al., 1993; Sands et al., 2008) e gli studi sul web management (Riegner, 2007; Fosdick, 2012) evidenzino la sua importanza crescente. In particolare, negli anni recenti, l'emergere del Web 2.0 ha influenzato le modalità con cui attori - come le imprese, gli utenti, i consumatori - interagiscono e cooperano durante lo scambio di informazioni e di conoscenza (Enders et al., 2008).

Constantinides e Fountain (2008) affermano che le più importanti applicazioni Web 2.0 (Social Network, Community, Blog) permettono di espandere l'esperienza, la conoscenza e il potere di mercato degli utenti. I Social Network vengono, inoltre, visti come strumenti in grado di migliorare la comunicazione (Berthon et al., 2012; Wang et al., 2012) e le relazioni con i clienti, la reputazione aziendale (Hawn, 2009) e la capacità di produrre innovazioni di prodotto/servizio (Kaplan e Haenlein, 2010) e di business networking. In particolare, i moderni Social consentono lo sviluppo di relazioni interattive tra aziende e utenti, offrendo opzioni aggiuntive alle imprese per l'aggiornamento di attività di marketing, come la promozione/ comunicazione, le vendite e le ricerche di mercato (Kaplan e Haenlein, 2010; Chen et al., 2011; Kirtis e Karahan, 2011; Berthon et al., 2012).

In considerazione di ciò, il presente studio si sofferma in particolare sull'analisi della dimensione sociale/interattiva della WA, cercando di proporre un contributo aggiuntivo al dibattito sul tema, pur restando focalizzato sulla gestione complessiva del sito web che continua ad essere il più importante generatore di contenuti veicolati attraverso le diverse applicazioni Web (tra cui i Social Media) (Istat, 2014).

\section{Metodo di ricerca e caratteristiche degli intervistati}

L'indagine empirica è stata finalizzata al duplice scopo della ricerca:

- testare la coerenza del framework di atmospherics proposto per i centri commerciali;

- analizzare gli elementi della WA che impattano maggiormente sugli utenti, in funzione del diverso atteggiamento che essi hanno nei confronti del centro commerciale.

Il metodo di ricerca si è basato sulla somministrazione di un questionario ad un campione di studenti iscritti all'Università di Urbino.

La scelta di focalizzarsi sui giovani studenti trova diverse giustificazioni. Mentre in passato gli studi sul comportamento degli individui negli shopping center hanno considerato principalmente gli adulti, un numero crescente di ricerche rileva che il segmento degli studenti rappresenta un mercato molto interessante per tale format. Studi nazionali (Cnr, 2009; De Fiori et al., 2010; Istat, 2014) ed internazionali supportano la rilevanza di questo target per i centri commerciali (Baker e Haytko, 2000; Matthews et al., 2000; Sari et al., 2015) e sempre più autori vi hanno focalizzato l'analisi (Haykto e Baker, 2004; Martin e Turley, 2004; El-Adly, 2007; Khaola et al., 2014; Sari et al., 2015).

Lattenzione verso i giovani, in generale, deriva anche dalla considerazione che questo target è potenzialmente interessato al centro commerciale in quanto più attratto dalla dimensione edonistica e ricreativa dello shopping 
sinergie Vol. 34, N. 100, 2016

di quanto lo siano gli adulti e gli anziani (Arnold e Reynolds, 2003; Martin e Turley, 2004; Massicote et al., 2011; Khaola et al., 2014). Il centro commerciale può rappresentare, infatti, un'interessante fonte di richiamo per tale segmento essendo un luogo di passatempo e socializzazione.

Oltre a queste considerazioni, la scelta di esaminare i giovani studenti, in questo lavoro, si lega al tecnicismo di alcune variabili utilizzate per descrivere la WA. In linea con altri studi sulla qualità e il design del sito web (Chen e Wells, 1999; Lin e Lu, 2000; Loiacono et al., 2007), si è ritenuto opportuno selezionare un campione che fa un uso rilevante e consapevole del Web (Rapporto Censis/UCSI, 2013) in modo da essere in grado di comprendere la terminologia e le problematiche essenziali connesse con le componenti e le caratteristiche del sito.

Il questionario è stato strutturato in tre sezioni:

- dati anagrafici;

- intensità di frequenza del centro commerciale e principali elementi di attrazione;

- rilevazione dell'importanza attribuita alle variabili della WA.

Le domande relative agli elementi di attrazione del centro commerciale ed agli attributi della WA di un ipotetico centro commerciale sono state sottoposte alla valutazione degli studenti mediante scala Likert a 5 passi.

In fase di elaborazione, per verificare la coerenza della WA in riferimento alle dimensioni individuate ed ai rispettivi attributi, si è proceduto, dapprima, all’analisi di asimmetria e curtosi; il test è utile per verificare se le risposte date hanno una distribuzione prossima o meno alla gaussiana. Da tale analisi risulta che gli indici di asimmetria e curtosi hanno valori sostanzialmente compresi tra $-1 \mathrm{e}+1$ per tutti gli attributi, perciò nessuno di questi può essere escluso.

Successivamente è stata effettuata unanalisi delle correlazioni e si è calcolato il coefficiente Alpha di Cronbach. Lobiettivo dell'analisi non è stato quello di verificare l'unidimensionalità del modello, quanto piuttosto la sua coerenza. A tal fine, ciascuna dimensione è stata ulteriormente testata attraverso un'analisi fattoriale basata sul metodo di estrazione delle componenti principali (Joliffe, 2002).

Successivamente, gli intervistati sono stati raggruppati in quattro segmenti, utilizzando come variabili discriminanti l'intensità di frequenza dei centri commerciali e gli elementi del format che esercitano la maggiore attrazione su di essi.

Nell'analizzare l'atteggiamento dei singoli segmenti rispetto alle variabili della WA, è stato effettuato il test sulle differenze tra le medie (T di Student) rispetto ad alcuni filtri importanti: maschi/femmine, scarsa/ elevata frequenza, atteggiamento funzionale/ricreativo.

Il campione è composto da 438 studenti. Il $67 \%$ degli intervistati ha un'età compresa tra 18 e 20 anni. La maggior parte degli intervistati è di sesso femminile (66\%) ed ha un profilo social (96\%). Il 59\% degli intervistati utilizza Internet mediamente da 1 a 3 ore al giorno; il 19\% da 3 a 5 ore.

Una percentuale molto esigua di intervistati $(3,65 \%)$ non frequenta i centri commerciali. Il $23,74 \%$ vi si reca solo occasionalmente (meno di una volta al mese). La maggior parte (50\%) dichiara di visitarli, in media, 
almeno 1 volta al mese, mentre i restanti si recano in tali strutture almeno 1 volta a settimana $(22,61 \%)$.

Gli elementi di maggiore attrazione del format sono nell'ordine: ampiezza dei prodotti/servizi offerti (136), presenza di offerte promozionali (132), presenza di attività di intrattenimento come cinema, ristoranti, spettacoli (64), possibilità di incontro con altre persone $(17)^{2}$. Sebbene l'offerta di intrattenimento sia stata valutata complessivamente importante, l'analisi dei dati rivela un atteggiamento di maggiore interesse nei confronti di quegli attributi riguardanti la varietà assortimentale e la convenienza economica.

Da rilevare la differenza delle valutazioni medie tra maschi e femmine soprattutto rispetto alla variabile "offerte promozionali": la media dei maschi è 3,60 mentre quella delle femmine è 3,87, delineando una maggiore attenzione di queste ultime nei confronti delle promozioni proposte dal centro commerciale.

\section{Analisi e discussione dei risultati}

\subsection{Analisi degli attributi della WA}

La lista di attributi descritti in Tab.1 è stata generata a partire da un riesame della letteratura esistente e da un'indagine svolta su un focus group tesa ad esplorare gli attributi su cui si dovrebbe basare la progettazione del sito web di un centro commerciale, affinché esso possa comunicare al meglio la dimensione esperienziale del format ed attrarre, in questo modo, visitatori al punto vendita fisico. Al focus hanno partecipato studenti universitari, aventi caratteristiche il più possibile simili a coloro che avrebbero successivamente risposto al questionario. La lista di attributi così ottenuta è stata valutata con metodo Delphi, mediante tre round di valutazioni effettuate con tre studenti dottorandi: gli attributi sono stati valutati sulla base di alcuni aspetti, quali la leggibilità, la chiarezza e la ridondanza, ed in base alla percezione di coerenza rispetto al costrutto oggetto di indagine. Al termine del processo sono rimaste le 35 variabili descritte in Tab.1.

Per valutare la coerenza interna del framework, è stata svolta, anzitutto, un'analisi di correlazione item-total. Nel caso di VLAY, VTHE e VSOC, i valori delle correlazioni interne sono decisamente maggiori rispetto a quelli rapportati alle altre variabili del framework; ciò supporta la validità della costruzione. Solo nel caso di VATM queste correlazioni sono meno evidenti: tale dimensione sembra essere meno supportata dai dati esaminati, anche se ciò non è sufficiente per giudicare la sua rappresentatività (Fig. 1).

2 Latteggiamento del consumatore nei confronti del centro commerciale dipende dal modo in cui esso si rapporta alla più generale attività di shopping. Esso può essere guidato da motivazioni di natura funzionale (Babin et al., 1994; Kim, 2006) oppure edonistica/ricreativa (Westbrook e Black, 1985; Babin et al., 1994). Contestualizzando l'analisi al centro commerciale, l'atteggiamento funzionale può tradursi nella ricerca di ampi assortimenti di prodotti/servizi e prezzi convenienti/offerte promozionali. L'atteggiamento edonistico/ricreativo, invece, può tradursi nella ricerca di intrattenimento e di momenti di socialità, indipendentemente dall'acquisto di prodotti e servizi (Arnold e Reynolds, 2003).
Marco Cioppi Elisabetta Savelli

Federica Tombari

La percezione della web
atmosphere atmosphere tra i visitatori del centro commerciale 
sinergie

Vol. 34, N. 100, 2016
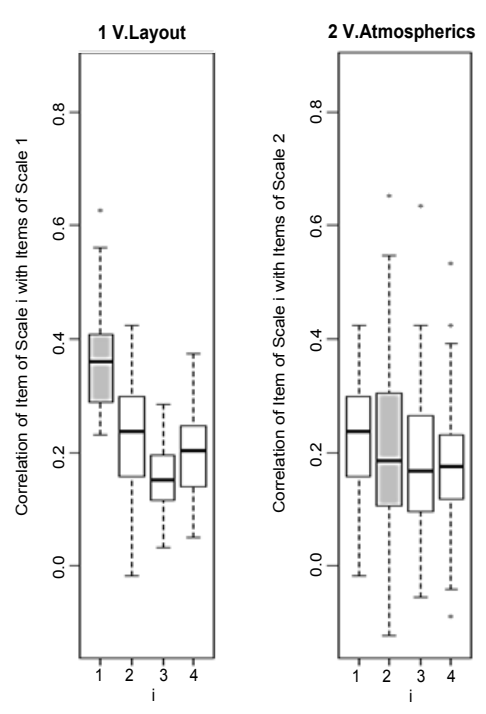

Fig.1: Item-total correlation

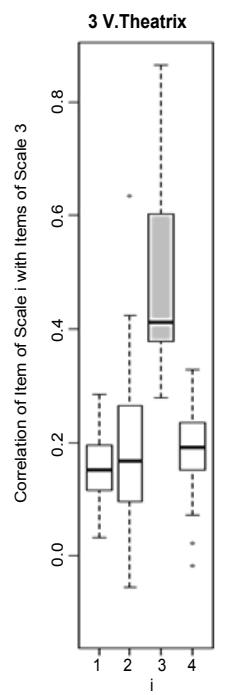

Fonte: ns elaborazione

Il calcolo del coefficiente Alpha di Cronbach supporta l'analisi itemtotal, rilevando valori superiori a quello di soglia $(>0.7)$ per tutte le dimensioni. Nel dettaglio: VLAY $=0.7760$, VATM $=0.7650$, VTHE $=0.8489$, VSOC $=0.8212$.

Ulteriore conferma della coerenza del framework è stata ricercata attraverso lo studio delle correlazioni interne ed una factor analysis esplorativa condotta su ciascuna dimensione (Joliffe, 2002). Di seguito, si riportano i risultati sintetici dell'analisi:

- VLAY: Lanalisi delle componenti principali conferma la robustezza della dimensione. Il grafico circolare delle correlazioni rileva che tutti gli attributi sono orientati nella stessa direzione (ovvero hanno correlazioni positive), sebbene alcuni di essi mostrino valori di correlazione più forti (Fig. 2), che potrebbero identificare l'esistenza di due sotto-dimensioni: VLAY1=(G01, G04, G05) comprendente variabili maggiormente attinenti la funzionalità nell'utilizzo del sito web, VLAY2 $=(\mathrm{G} 02, \mathrm{G} 03, \mathrm{G} 06)$ comprendente variabili che riguardano la funzionalità nella ricerca dei contenuti web. Questa prima dimensione è comunque robusta e coerente. 
Fig. 2: Scree plot e item correlation (VLAY)
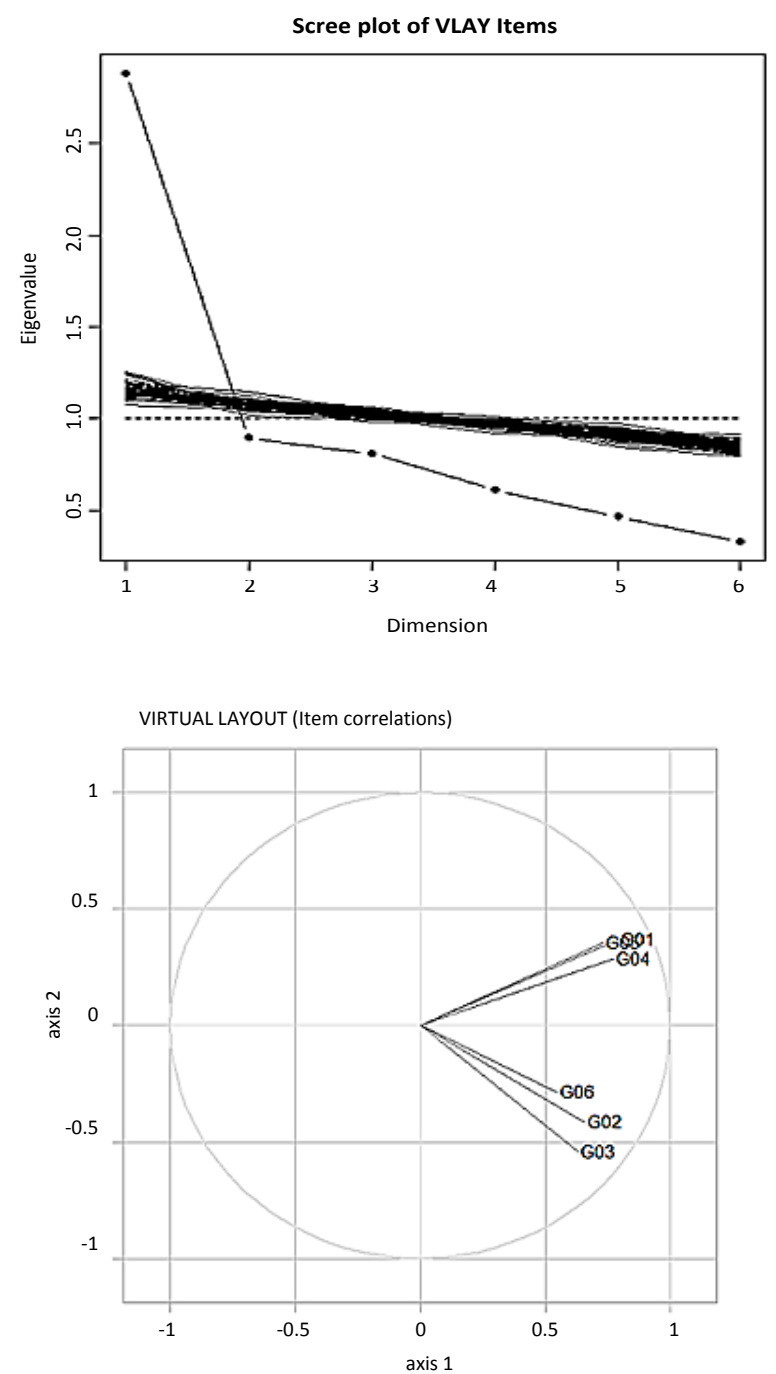

Marco Cioppi Elisabetta Savell

Federica Tombari

La percezione della web atmosphere

tra i visitatori del centro commerciale

Fonte: ns elaborazione

- VATM: Lanalisi delle componenti principali non conferma pienamente la robustezza della dimensione, anche se ciò non compromette la sua coerenza.

Il grafico circolare delle correlazioni conferma la positività di queste ultime (Fig. 3), sebbene si possa notare una differente intensità di correlazione tra attributi che potrebbe identificare alcune sottodimensioni (VATM1 $=(\mathrm{G} 07, \mathrm{G} 08, \mathrm{G} 11, \mathrm{G} 12, \mathrm{G} 16)$, VATM2 $=(\mathrm{G} 09, \mathrm{G} 10$, G14, G18), VATM3 $=(\mathrm{G} 13, \mathrm{G} 15, \mathrm{G} 17))$, le quali tuttavia, non appaiono nitide nemmeno sotto il profilo concettuale. In definitiva, questa dimensione pur essendo ben rappresentata, si può considerare più debole delle altre. 
sinergie

Vol. 34, N. 100, 2016
Fig. 3: Scree plot e item correlation (VATM)

Scree plot of VATM Items

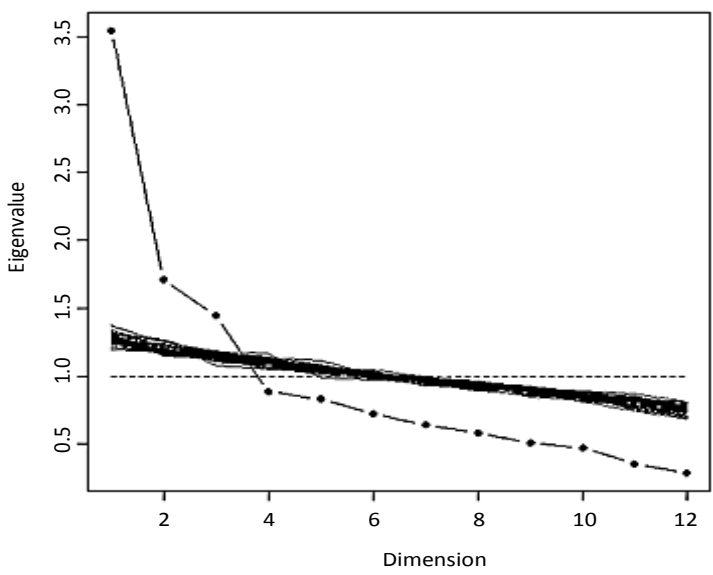

VIRTUAL ATHMOSPHERICS (Item correlations)

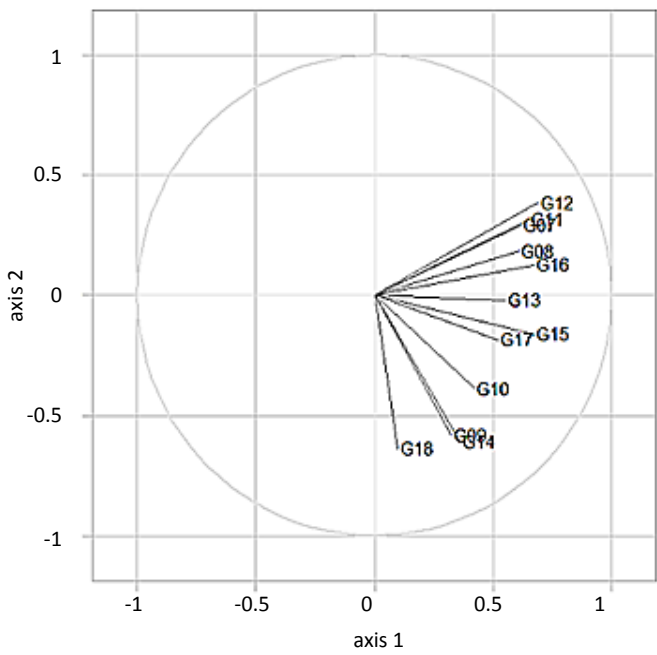

Fonte: ns elaborazione

- VTHE: Lanalisi delle componenti principali conferma la robustezza della dimensione. Il grafico circolare delle correlazioni mette in rilievo che tutti gli attributi sono orientati nella stessa direzione (identificando correlazioni positive), sebbene alcuni abbiano un grado di correlazione più forte rispetto ad altri (Fig.4). Come per la prima dimensione, si potrebbero identificare due sotto-dimensioni: VTHE1=(G19, G24), attinente gli aspetti visivi del sito web che richiedono interattività dell'utente, e VTHE2=(G20, G21, G22, G23), attinente gli attributi visivi che presuppongono un atteggiamento dell'utente più passivo. Nell'insieme, anche questa dimensione è robusta e coerente. 
Fig. 4: Scree plot e item correlation (VTHE)
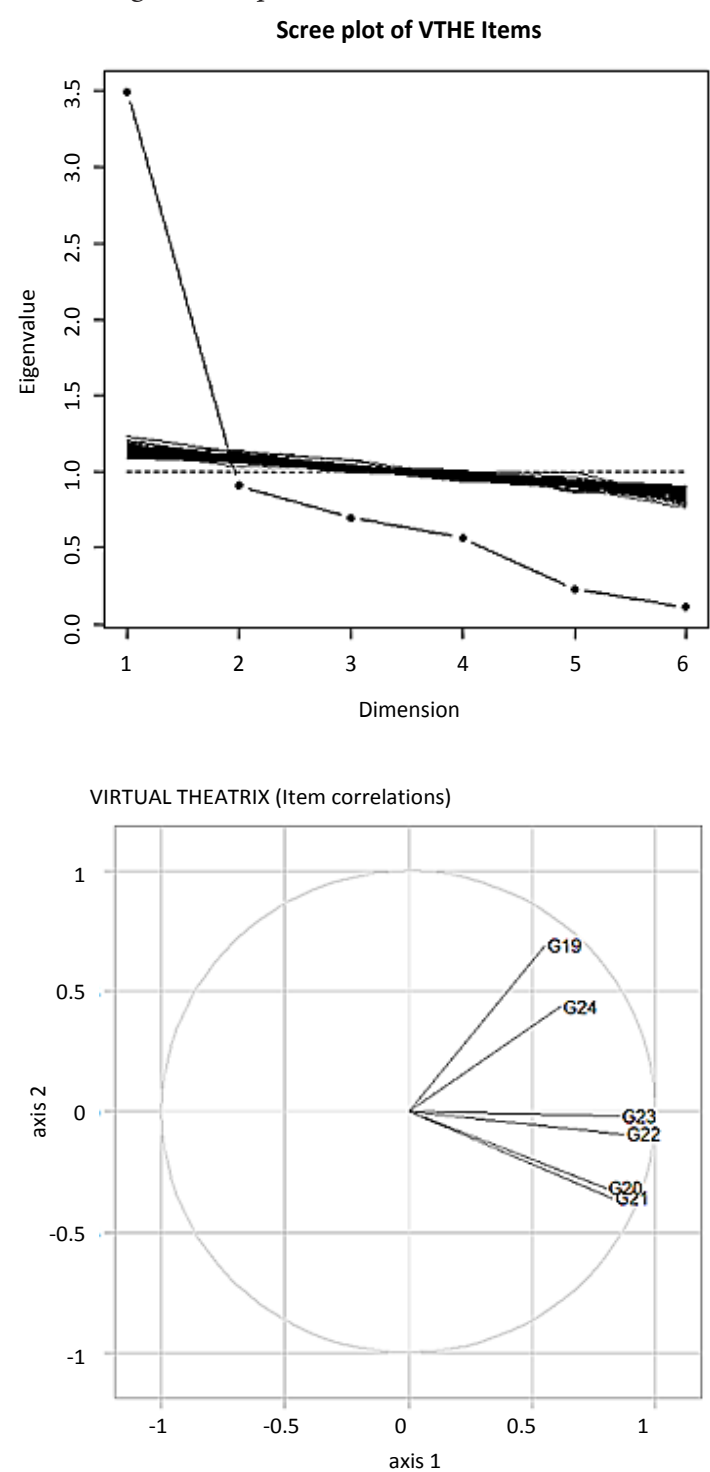

Fonte: ns elaborazione

- VSOC: L'analisi delle componenti principali conferma la robustezza della dimensione. Il grafico circolare delle correlazioni mette in rilievo che tutti gli attributi sono ugualmente orientati (quindi hanno correlazioni positive) e sono ben raggruppati tra loro ad esclusione di uno (G35) (Fig.5). Anche in quest'ultimo caso si potrebbero identificare due sottodimensioni: VSOC1 $=(G 25, G 26, G 27, G 28, G 29, G 30)$ e VSOC2 $=(G 31$, G32, G33, G34, G35), attinenti rispettivamente gli attributi di comunicazione tipici del Web 2.0 (di natura più social) e quelli tipici del Web 1.0 (telefono, e-mail, ecc.). 
sinergie

Vol. 34, N. 100, 2016
Fig. 5: Scree plot e item correlation (VSOC)

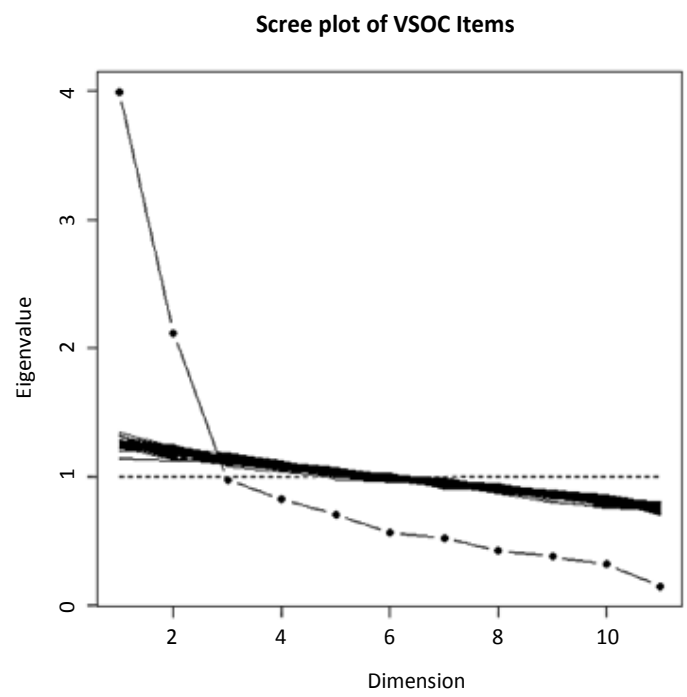

VIRTUAL SOCIAL PRESENCE (Item correlations)

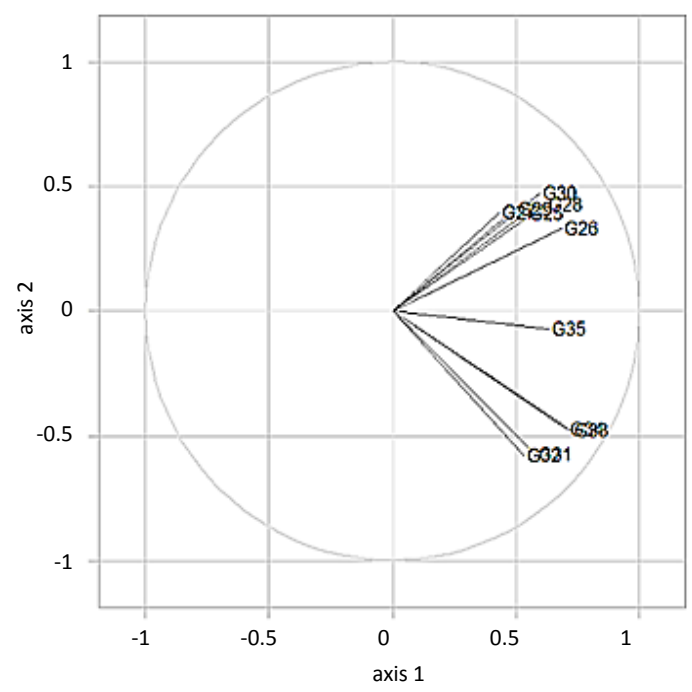

Fonte: ns elaborazione

Le analisi confermano, in definitiva, la coerenza interna del framework di atmospherics. Le correlazioni sono positive e l'Alpha di Cronbach è sempre superiore al valore di soglia $(>0.7)$. Lanalisi delle componenti principali non permette di dimostrare l'esistenza di una scala perfettamente unidimensionale ma questo non significa che il framework non sia rappresentativo e coerente. I grafici circolari delle correlazioni dimostrano, infatti, che quasi tutte le dimensioni sono ben rappresentate, sebbene si potrebbero individuare alcune sotto-dimensioni per rendere più robusto il modello. 
Dopo aver analizzato la coerenza del framework, si è approfondito il medio di visite al centro commerciale e degli elementi del format che esercitano la maggiore attrazione su di essi.

Si è stabilito che coloro che si recano in tali strutture almeno una volta al mese o almeno una volta a settimana appartengono alla categoria "High Frequency", mentre coloro che vi si recano meno di una volta al mese o mai appartengono al gruppo "Low Frequency". Raggruppando così gli intervistati, il $72,61 \%$ del totale visita con discreta regolarità il centro commerciale: da un minimo di una volta al mese a un massimo di una o più volte a settimana. Il restante $27,39 \%$ dichiara di recarvisi meno di 1 volta al mese oppure mai. Questi dati sono in linea con precedenti studi che evidenziano l'importanza del target giovanile per il format in considerazione (cfr. par. 3).

Anche gli elementi di attrazione del format sono stati raggruppati in due categorie ${ }^{3}$ :

- elementi funzionali: comprendono l'ampiezza dei prodotti/servizi offerti

(F1) e la presenza di offerte promozionali (F2);

- elementi ricreativi: comprendono l'offerta di attività di intrattenimento

(F3) e la possibilità di incontrare altre persone (F4).

In generale, $\mathrm{i}$ frequentatori più assidui tendono ad attribuire un punteggio mediamente più alto a tutti gli elementi di attrazione considerati; i visitatori meno frequenti, invece, essendo già di partenza meno attratti dal format, attribuiscono valori più cauti ai singoli elementi indagati (Tab.2). In entrambi i casi, le dimensioni del centro commerciale più interessanti risultano essere quelle di natura funzionale. Rilevante è anche la valutazione positiva del fattore intrattenimento da parte dei frequentatori più assidui. Rispetto agli altri, questi ultimi tendono ad attribuire un valore mediamente superiore alle caratteristiche afferenti la dimensione ricreativo/esperienziale del centro.

Tab. 2: Elementi di attrazione del centro commerciale: confronto tra visitatori con scarsa o medio/alta frequenza

\begin{tabular}{lcccccccc}
\hline & Low Frequency & & \multicolumn{5}{c}{ High Frequency } \\
& $\begin{array}{c}\text { Valore } \\
<3\end{array}$ & $\begin{array}{c}\text { Valore } \\
=3\end{array}$ & $\begin{array}{c}\text { Valore } \\
>3\end{array}$ & $\begin{array}{c}\text { Valore } \\
\text { medio }\end{array}$ & $\begin{array}{c}\text { Valore } \\
<3\end{array}$ & $\begin{array}{l}\text { Valore } \\
=3\end{array}$ & $\begin{array}{l}\text { Valore } \\
>3\end{array}$ & $\begin{array}{l}\text { Valore } \\
\text { medio }\end{array}$ \\
\hline $\begin{array}{l}\text { ampiezza } \\
\text { prodotti/servizi }\end{array}$ & 20 & 24 & 76 & 3,73 & 41 & 69 & 208 & 3,82 \\
\hline offerte promozionali & 29 & 19 & 72 & 3,62 & 32 & 74 & 212 & 3,84 \\
\hline attività di intrattenimento & 54 & 27 & 39 & 2,78 & 102 & 81 & 135 & 3,18 \\
\hline $\begin{array}{l}\text { possibilità di incontro } \\
\text { con altre persone }\end{array}$ & 79 & 24 & 17 & 2,15 & 181 & 82 & 55 & 2,37 \\
\hline
\end{tabular}

Fonte: ns elaborazione

3 Cfr. precedente nota 2. 


\section{sinergie} Vol. 34, N. 100, 2016

Considerando il test sulle differenze tra le medie, il valore è statisticamente irrilevante per la prima variabile (ampiezza prodotti e servizi offerti), mentre risulta importante per gli altri fattori di attrazione considerati: i visitatori High Freqency danno maggiore rilievo, rispetto a quelli meno frequenti, a queste ultime tre variabili.

Approfondendo il confronto tra l'intensità di frequenza del centro commerciale e gli elementi di attrazione del format si è tentato di delineare una possibile segmentazione dei visitatori.

Poiché i diversi fattori di attrazione sono stati valutati con scala Likert a 5 passi, si è assunto che la singola categoria di elementi (funzionali e ricreativi) è significativa quando il valore attribuito contemporaneamente ai due fattori è maggiore di 3 .

Prendendo le categorie-limite, composte dai soli soggetti che attribuiscono esclusiva importanza alla dimensione funzionale oppure a quella ricreativa, e considerando la ripartizione del campione tra Low e High Frequency, sono stati individuati i seguenti segmenti : cacciatori di offerte (25\%), distensivi (3\%), funzionali (65\%), ricreativi (7\%).

I "cacciatori di offerte" visitano con scarsa frequenza il centro commerciale ed attribuiscono esclusiva importanza alla dimensione funzionale/economica del format: prediligono la varietà assortimentale e vi si recano prevalentemente in occasione di offerte promozionali giudicate allettanti.

I "distensivi" visitano saltuariamente il centro commerciale ma sono più attratti dagli elementi ricreativi. Può essere il caso dell'utente che si reca al centro commerciale solo perché attratto, per esempio, da un determinato evento.

"Funzionali" e "ricreativi" visitano con regolarità abbastanza intensa il centro commerciale: i primi sono attratti dallefficienza funzionale e sono costantemente attenti a sfruttare le offerte promozionali proposte; i "ricreativi" sono coloro che apprezzano esclusivamente la capacità attrattiva del centro commerciale legata allofferta di attività ludiche e di intrattenimento e sono attirati a frequentare i servizi ricreativi presenti al suo interno.

\subsection{Tipologie di utenti e atteggiamento nei confronti della WA}

I "cacciatori di offerte" e i "funzionali" attribuiscono maggiore importanza, rispetto alla media, alle variabili attinenti la navigabilità del sito web (VLAY). Si presume che questi soggetti, essendo molto attenti alle offerte promozionali del centro, visitino il sito web esclusivamente per documentarsi sulla presenza di eventuali iniziative. Ne consegue una naturale attenzione alle dimensioni riguardanti la funzionalità e l'usabilità del sito. A riprova di ciò, si evidenzia un atteggiamento dei "ricreativi" connotato da una scarsa attenzione alle suddette variabili.

In relazione a VATM, "cacciatori di offerte", "funzionali" e "ricreativi" attribuiscono valori mediamente superiori rispetto al campione. I "funzionali", in particolare, sono molto attenti alle informazioni riguardanti le carte promozionali attivate dal centro. "Distensivi" e "ricreativi" sono più 
attenti alla presenza di banner pubblicitari, all'audio del sito ed alla presenza di informazioni aggiornate sugli eventi e le attività d'intrattenimento organizzate dal centro. Ciò riflette il diverso atteggiamento delle due tipologie di utenti: da un lato, i "cacciatori di offerte" e i "funzionali" che, in generale, sono più attenti alle informazioni di carattere economico/commerciale; dall'altro, gli individui attratti dalla dimensione ludico/ricreativa del centro commerciale che focalizzano maggiormente l'attenzione sulle informazioni in grado di veicolare questo tipo di offerta.

Rispetto alle variabili VTHE, i "cacciatori di offerte" e i "distensivi" mostrano un atteggiamento di minore interesse. La scarsa frequenza con cui visitano il centro commerciale potrebbe riflettersi anche in una scarsa frequenza di accesso ai relativi siti web che vengono visitati prestando esclusiva attenzione al contenuto informativo di carattere promo/ commerciale, trascurando completamente il suo aspetto più teatrale.

Per quanto riguarda, infine, la dimensione VSOC, i "funzionali" e i "ricreativi" sono mediamente più attenti a queste variabili rispetto ai "cacciatori di offerte" e i "distensivi". Anche questo riflette il diverso atteggiamento degli utenti: "funzionali e "ricreativi" visitano con più frequenza i centri commerciali e tendono ad attribuire maggiore importanza allo sviluppo di relazioni di lungo termine, finalizzate alla fruizione di informazioni e contenuti ricchi ed approfonditi.

La conduzione di ulteriori test sulle differenze tra le medie rileva considerazioni aggiuntive che confermano quanto detto.

I frequentatori dei centri commerciali (rispetto ai non frequentatori) tendono ad attribuire un punteggio mediamente più alto a tutti gli attributi della WA. Le differenze più rilevanti si riscontrano in riferimento ad alcune variabili di VLAY e VATM che sono più interessanti per chi visita regolarmente tali strutture (accessibilità: 3.66 vs. 3.52, velocità: 4.10 vs. 3.89, informazioni su carte promozionali: 3.21 vs. 2.88 ; disponibilità di informazioni aggiornate: 3.75 vs. 3.58 , presenza di banner pubblicitari relativi al centro commerciale: 2.42 vs. 2.21 ) ed agli attributi visivi (VTHE) basati sulla presenza di immagini (photogallery del centro: 3.57 vs. 3.29; photogallery dei singoli negozi: 3.53 vs. 3.31 ).

Altre differenze si rilevano in relazione all'atteggiamento verso il centro commerciale. I funzionali, esprimono valutazioni mediamente più elevate in riferimento a tutte le variabili VLAY attinenti l'aspetto funzionale del sito ed a tutte le variabili attinenti il contenuto informativo (VATM), ad eccezione di quella riguardante le informazioni sugli eventi organizzati dal centro (evidentemente i "funzionali" sono meno interessati a ciò). Inoltre, si riscontrano differenze in relazione alle variabili VSOC, ad eccezione di quelle relative alla presenza di live chat e forum, probabilmente perché i funzionali sono anche meno attratti dalla dimensione sociale del format. I ricreativi, invece, valutano con maggiore importanza gli attributi inerenti la velocità di navigazione ( 3.90 vs. 3.72$)$, la presenza di link ad altri siti (3.20 vs. 2.84$)$ e il fatto che il sito sia multilingue (3.57 vs. 3.03$)$. Inoltre, si rilevano differenze particolari in merito ai seguenti attributi: disponibilità di informazioni sul centro commerciale ( 3.48 vs. 3.20 ), informazioni su eventi (3.42 vs. 2.60 ) e sfondo audio (2.28 vs. 1.83 ), per quanto riguarda la dimensione VATM; forum di discussione (2.68 vs. 2.33), link ai social
Marco Cioppi

Elisabetta Savelli

La percezione della web

atmosphere

tra i visitatori del centro commerciale 
sinergie Vol. 34, N. 100, 2016

network (3.06 vs. 2.57), presenza di live chat (2.36 vs. 2.01$)$ e modulo di richiesta informazioni online (2.96 vs. 2.57), in relazione alla dimensione VSOC. Tutto ciò testimonia e rafforza che l'atteggiamento di shopping degli utenti si riflette anche sulle modalità di navigazione.

\section{Conclusioni}

Lanalisi svolta fornisce una risposta affermativa al quesito iniziale della ricerca: l'atteggiamento degli utenti nei confronti del centro commerciale influenza le finalità per cui il sito web viene utilizzato; nello stesso tempo, a prescindere dall'orientamento dell'utente - funzionale o ricreativo - i giovani intervistati apprezzano in maniera particolare la presenza di attributi che favoriscono la comunicazione e l'interattività. Tale atteggiamento tende ad essere tanto più forte quanto più i consumatori frequentano con regolarità il centro commerciale. Questi, infatti, attribuiscono maggiore importanza al contenuto esperienziale del format; di conseguenza, ripongono maggiore attenzione alle variabili del sito web riguardanti la dimensione sociale ed interattiva.

Ciò mette in luce alcuni elementi che fanno riflettere sulle implicazioni teoriche e manageriali derivanti dalla ricerca.

Sotto il profilo teorico, lo studio fornisce indicazioni sia metodologiche che concettuali.

In termini metodologici, il principale apporto è legato allo svolgimento di unanalisi completa della WA, basato su un framework di attributi più articolato rispetto ai modelli proposti in passato. La maggior parte delle ricerche precedenti, infatti, si sono soffermate sull'analisi di singoli atmospherics, quali la musica (Kim e Lennon, 2012), i colori (Koo e Ju, 2010) o il disegno di navigazione (Taylor e England, 2006). Lo studio, inoltre, propone un framework di attributi applicato ad uno specifico contesto aziendale, quello dei centri commerciali, seguendo l'indicazione di Tordjman (1994) secondo cui l'importanza degli atmospherics dipende dalla peculiarità dei settori analizzati. Il lavoro contribuisce, dunque, alla letteratura esistente sul retail management, individuando un framework di variabili in grado di cogliere le specificità del contesto indagato.

In termini concettuali, lo studio rafforza i risultati di precedenti ricerche che sottolineano l'importanza degli attributi sociali e relazionali del sito web, capaci di esaltarne l'interattività (Baloglu e Peckan, 2006; Riegner, 2007; Fosdick, 2012). Il lavoro sottolinea, infatti, il ruolo critico della dimensione VSOC nella valutazione del sito web da parte degli utenti.

Anche sotto il profilo manageriale, l'analisi suggerisce implicazioni interessanti.

Anzitutto, il valore mediamente alto attribuito agli elementi della WA (soprattutto da parte dei frequentatori più assidui del centro commerciale) raccomanda la necessità di sviluppare competenze organizzative in grado di valorizzare la comunicazione esperienziale online. Ciò richiede sforzi adeguati in termini di contenuti: occorre inserire informazioni e strumenti in grado di favorire l'interazione e lo scambio di comunicazione (Huotari et al., 2015), per esempio attraverso la creazione di comunità virtuali, blog 
di discussione e collegamenti con profili social che aumentano la capacità del sito web di svolgere un ruolo di aggregatore di utenti. Ancora, investimenti mirati a rafforzare la presenza e le promozioni sui social network e sugli altri strumenti di comunicazione interattiva potrebbero essere utili per incrementare le visite del sito per motivi di svago ed intrattenimento e il livello di fidelizzazione degli utenti. Inoltre è richiesto un grande impegno sotto il profilo manageriale. La gestione di un forum, di una live chat o di una pagina social necessita, infatti, di risorse preparate (Andzulis et al., 2012), capaci di interagire in qualunque momento con l'utente e di fornirgli informazioni rapide e sempre aggiornate. Eventuali disservizi su questo fronte potrebbero originare fenomeni di passaparola negativi dannosi per l'immagine e il brand aziendale.

Il risultato della ricerca che rivela l'esistenza di differenti tipologie di utenti, dai cacciatori di offerte ai ricreativi, con specifiche peculiarità ed esigenze comunicative, suggerisce una seconda implicazione. La comunicazione online del centro commerciale non può essere standardizzata, ma disegnata nella struttura delle informazioni e nei contenuti in funzione delle specifiche esigenze del target al quale l'impresa ha deciso di indirizzarsi. Oggigiorno esistono numerosi strumenti che permettono al sito web di essere responsive ai devices dell'utente ed offrono la possibilità di usufruire di informazioni e servizi on demand. Basta dotare il sito di cookie, pixel tag, strumenti di analisi del browser, log del server ed altre tecnologie simili per poter raccogliere una grande quantità di informazioni sui visitatori e il loro comportamento in rete. Il problema, tuttavia, non è solo tecnico: gli strumenti automatici di profilazione dell'utenza sono ormai alla portata di tutti e questi permettono di personalizzare i contenuti adattandoli ai singoli casi. È sotto il profilo strategico che il management dovrebbe compiere una precisa scelta di target ing in funzione della quale deve essere progettata sia la struttura commerciale che quella del sito web. La comunicazione online, in altre parole, dovrebbe essere realizzata in funzione delle specifiche esigenze non solo dell'utente in rete, ma, soprattutto, dell'utente-target al quale il management ha deciso di indirizzarsi.

Una terza implicazione scaturisce dalla considerazione che il $65 \%$ del campione analizzato appartiene alla categoria dei funzionali. I giovani intervistati sono attratti soprattutto dall'efficienza funzionale del format. Se questo tende ad essere l'atteggiamento prevalente tra i giovani, assume rilevanza la capacità di sviluppare sistematiche e coordinate iniziative promozionali da veicolare attraverso il sito, che dovrebbe essere continuamente aggiornato per dare la possibilità agli utenti di informarsi in tempo reale sulle iniziative attivate dal punto vendita. In altre parole, il sito web dovrebbe essere utilizzato diffusamente come strumento di promozione, anche riservando delle specifiche offerte agli utenti in rete, oppure ricorrendo a concorsi ed altre iniziative specifiche per coloro che navigano con regolarità il sito. Quest'ultimo potrebbe anche essere studiato per contenere applicazioni scaricabili su smartphone, che permettono all'utente di essere sempre aggiornato, anche in maniera personalizzata, sulle novità promozionali attivate dal centro commerciale. Lorientamento funzionale dell'utente si traduce anche in un atteggiamento di particolare interesse verso l'accessibilità sia esterna che interna al sito web. Sul piano 
sinergie Vol. 34, N. 100, 2016

della progettualità, ciò richiede una buona indicizzazione nei motori di ricerca e l'utilizzo di funzionalità di ricerca avanzate. In altre parole, il design dovrebbe essere concepito in maniera altrettanto efficace quanto i contenuti, per soddisfare al meglio le esigenze di funzionalità dell'utente.

Anche ciò evidenzia l'importanza di strutturare risorse specializzate nella comunicazione, nell'aggiornamento dei dati e nell'interazione con l'utenza. La gestione del sito, anche qualora venga concepito come semplice vetrina informativa (cosa frequente tra i centri commerciali), non può essere destrutturata e casuale: occorrono risorse, competenze e soprattutto una pianificazione adeguata delle attività, coerente con il resto della comunicazione aziendale e, ancor più in generale, con la strategia di mercato che l'impresa intende perseguire.

I risultati presentati in questo lavoro rappresentano il punto di partenza di un progetto più ampio che prevede ulteriori elaborazioni ed un'estensione dell'analisi.

Anzitutto, considerando il framework di attributi proposto, le analisi statistiche preliminari dimostrano una buona coerenza interna e suggeriscono di approfondire la validazione del framework al fine di individuare una vera e propria scala di attributi riferita al contesto dei centri commerciali.

In secondo luogo, il campione scelto in questo lavoro è composto da studenti universitari che hanno uno stile di vita particolare e comune. Sebbene il target giovanile sia potenzialmente interessante per il centro commerciale, come dimostrato in studi passati ed ulteriormente rilevato nella presente analisi, è interesse degli autori estendere, in futuro, la ricerca ad un campione più ampio di potenziali utenti per verificare l'effettiva consistenza dei segmenti proposti ed i relativi atteggiamenti nei confronti del sito web.

Infine, poiché in questo studio non sono state considerate le caratteristiche strutturali (dimensioni, tipologie di negozi e offerte proposte ai visitatori, età ed originalità della struttura...) e logistiche (vicinanza di grandi centri urbani, raggiungibilità,...) dei centri commerciali, sarebbe interessante approfondire in che modo questi attributi possono influenzare l'atteggiamento dell'utente nella valutazione della WA e le effettive modalità di gestione del sito web da parte delle imprese commerciali.

\section{Bibliografia}

AINSWORTH J., BALLANTINE P.W. (2014), "That's different! How consumers respond to retail websitechange", Journal of Retailing and Consumer Services, vol. 21, n. 5, pp. 764-772.

AHN T., SEEWON R., INGOO H. (2005), "The impact of the online and offline features on the user acceptance of Internet shopping malls", Electronic Commerce Research and Applications, vol. 3, n. 4, pp. 405-420.

ALADWANI A.M., PALVIA P.C. (2002), "Developing and validating an instrument for measuring user-perceived web quality", Information and Management, vol. 39, n. 6, pp. 467-476.

ANDZULIS, J.M., PANAGOPOULOS, N.G., RAPP, A. (2012), "A review of social media and implications for the sales process", Journal of Personal Selling 
and Sales Management, vol. 32, n. 3, pp. 305-316.

ARNOLD M.J., REYNOLDS K. (2003), "Hedonic Shopping Motivations", Journal of Federica Tombari Retailing, vol. 79, n. 2, pp. 77-95.

La percezione della web atmosphere

BABIN B.J., ATTAWAY J.S. (2000), "Atmospheric as Tool for Creating Value and commerciale ivisitatori del centro Gaining Share of Customer", Journal of Business Research, vol. 49, n. 2, pp. 91-99.

BABIN B.J., DARDEN W., GRIFFIN M. (1994), "Work and/or Fun: Measuring Hedonic and Utilitarian Shopping Value", Journal of Consumer Research, vol. 20, n. 4, pp. 644-656.

BAI B., LAW R., WEN I. (2008), "The impact of websitequality on customer satisfaction and purchase intentions: evidence from Chinese online visitors", International Journal of Hospitality Management, n. 27, pp. 391-402.

BAKER J., HAYTKO D. (2000), “The mall as entertainment: Exploring teen girls' total shopping experiences", Journal of Shopping Center Research, vol. 7, n. 1, pp. 29-58.

BAKER J., GREWAL D., PARASURAMAN A. (1993), "The Influence of Store Environment on Quality Inferences and Store Image", Journal of the Academy of Marketing Science, vol. 22, n. 4, pp. 328-339.

BALOGLU S., PECKAN Y.A. (2006), “The websitedesign and Internet site marketing practices of upscale and luxury hotels in Turkey", Tourism Management, vol. 27, n. 1, pp. 171-176.

BANERJEE D.N. (2012), "A Study on The Attractiveness Dimensions of Shopping Malls - An Indian Perspective", International Journal of Business and Social Science, vol. 3, n. 2, pp. 102-111.

BARLOW A.K., SIDDIQUI N.Q., MANNION M. (2004), "Developments in information and communication technologies for retail marketing channels", International Journal of Retail and Distribution Management, vol. 32, n. 3, pp. 157-163.

BERTHON P.R., PITT L.F., PLANGGER K., SHAPIRO D. (2012), "Marketing meets Web 2.0, social media, and creative consumers: Implications for international marketing strategy", Business Horizons, vol. 55, n. 3, pp. 261-271.

BHATTI N., BOUCH A., KUCHINSKY A. (2000), "Integrating user-perceived quality into web server design", Computer Networks, vol. 33, n. 1-6, pp. 1-16.

BELANCHE D., CASALÓ L.V., GUINALÍU M. (2012), "Websiteusability, consumer satisfaction and the intention to use a website: the moderating effect of perceived risk", Journal of Retailing and Consumer Services, vol. 19, n. 1, pp. 124-132.

BELLMAN S., LOHSE G.L., JOHNSON E.J. (1999), "Predictors of online buying: Findings from the Wharton virtual test market", Communications of the $A C M$, vol. 42, n. 12, pp. 32-28.

CAO M., ZHANG Q., SEYDEL J. (2005), "B2C e-commercewebsitequality: An empirical examination", Industrial Management and Data Systems, vol. 105, n. 5, pp. 645-661.

CHEN K., YEN D.C. (2004), "Improving the quality of online presence through interactivity", Information and Management, n. 42, pp. 217-226.

CHEN Q., WELLS W.D. (1999), "Attitude Toward the Site", Journal of Advertising Research, vol. 39, n. 5, pp. 27-38.

CHEN Y., FAY S., WANG Q. (2011), “The role of marketing in social media: How online consumer reviews evolve”, Journal of Interactive Marketing, vol. 25, n. 
sinergie Vol. 34, N. 100, 2016

2, pp. 85-94.

CHEN Y.H., HSU I., LIN C.C. (2010), "Websiteattributes that increase consumer purchase intention: A conjoint analysis", Journal of Business Research, vol. 63, n. 9, pp. 1007-1014.

CIOPPI M., SAVELLI E. (2014), "Atteggiamento degli utenti verso il centro commerciale e finalità di utilizzo del sito web", in Atti dell'XI Convegno Annuale della Società Italiana Marketing Food Marketing, Modena 18-19 settembre.

CNCC (2015), L'industria dei centri commerciali in Italia, Consiglio nazionale dei centri Commerciali, Milano.

CNR (2009), "Centro commercial batte museo e biblioteca", documento disponibile online: http://www.cnr.it/news/index/news/id/4816 (ultimo accesso: 2 marzo 2016).

CONSTANTINIDES E., FOUNTAIN S.J. (2008), "Web 2.0: Conceptual foundations and marketing issues", Journal of Direct, Data and Digital Marketing Practice, vol. 9, n. 3, pp. 231-244.

DAILEY L. (2004), "Navigational web atmospherics: explaining the influence of restrictive navigation cues", Journal of Business Research, vol. 57, n. 7, pp. 795-803.

DAWSON S., KIM. M. (2010), "Cues on Apparel Web Sites that Trigger Impulse Purchases", Journal of Fashion Marketing and Management, vol. 14, n. 2, pp. 230-246.

DE FIORI A., JACONO QUARANTINO M., LAZZARI M. (2010), "L'uso di strumenti di comunicazione telematica fra gli adolescenti", in Lazzari M., Jacono Quarantino M. (a cura di), Adolescenti tra piazze reali e piazze virtuali, Bergamo University Press.

DE NISCO A., NAPOLITANO M.R. (2006), "Entertainment Orientation of Italian Shopping centers: Antecedents and Performance", Managing Service Quality, vol. 16, n. 2, pp. 145-166.

DECK S. (1997), "Ease of navigation key to successful e-malls", Computerworld, vol. 31, n. 28, pp. 4 e ss.

DELONE W.H., MCLEAN E.R. (1992), "Information systems success: The quest for the dependent variable", Information Systems Research, vol. 3, n. 1, pp. 60-92.

DRÈZE X., ZUFRYDEN F. (2004), "Measurement of online visibility and its impact on Internet traffic", Journal of Interactive Marketing, vol. 18, n. 1, pp. 20-37.

EL-ADLY M.I. (2007), "Shopping malls attractiveness: a segmentation approach", International Journal of Retail and Distribution Management, vol. 35, n. 11, pp. 936-950.

ENDERS A., HUNGENBERG H., DENKER H.P., MAUCH S. (2008), "The long tail of social networking: Revenue models of social networking sites", European Management Journal, vol. 26, n. 3, pp. 199-211.

EROGLU S.A., MACHLEIT K.A., DAVIS L.M. (2003), "Empirical testing of a model of online store atmospherics and shopper responses", Psychology and Marketing, vol. 20, n. 2, pp. 139-150.

EUROSTAT (2014), "E-commerce statistics", Documento disponibile online: http://ec.europa.eu/eurostat/statistics-explained/index.php/E-commerce_ statistics (ultimo accesso: 4 marzo 2016).

FOSDICK M. (2012), “The globalization of social media: consumer relationships 
with brands evolve in the digital space", Strategic Direction, vol. 28, n. 6.

GALLUCCI F., POPONESSI P. (2008), Il marketing dei luoghi e delle emozioni, Egea, Milano.

GATAUTIS R., VAICIUKYNAITE E. (2013), "WebsiteAtmosphere: Towards Revisited Taxonomy Of WebsiteElements", Economics and Management, vol. 18, n. 3, pp. 537-544.

HAUSMAN A.V, SIEKPE J.S. (2009), "The effect of web interface features on consumer online purchase intentions", Journal of Business Research, vol. 62, n. 1, pp. 5-13.

HAWN C. (2009), “Take Two Aspirin and Tweet Me In The Morning: How Twitter, Facebook, and Other Social Media Are Reshaping Health Care", Health Affairs, vol. 28, n. 2, pp. 361-368.

HAYTKO D.L., BAKER J. (2004), "It's all at the mall: exploring adolescent girls' experiences", Journal of Retailing, vol. 80, n. 1, pp. 67-83.

HERNÁNDEZ B., JIMÉNEZ J., MARTÍN M.J. (2009), "Key websitefactors in e-business strategy", International Journal of Information Management, vol. 29 , n. 5, pp. 362-371.

HIRSCHMAN E.C., HOLBROOK M.B. (1982), "Hedonic Consumption: Emerging Concepts, Methods and Propositions", Journal of Marketing, vol. 46, n. 3, pp. 92-101.

HUOTARI L., ULKUNIEMI P., SARANIEMI S., MÄLÄSKÄ M. (2015), "Analysis of content creation in social media by B2B companies", Journal of Business and Industrial Marketing, vol. 30, n. 6, pp. 761-770.

JIANG Z., CHAN J., TAN B.C.Y., CHUA W.S. (2010), "Effects of interactivity on websiteinvolvement and purchase intention", Journal of the Association for Information Systems, vol. 11, n. 1.

JOLLIFFE I. (2002), Principal component analysis, John Wiley \& Sons, Ltd., New York.

KAPLAN A.M., HAENLEIN M. (2010), "Users of the world, unite! The challenges and opportunities of Social Media", Business Horizons, vol. 53, n. 1, pp. 59-68.

KHAOLA P.P., POTIANE B., MOKHETHI M. (2014), "Environmental concern, attitude towards green products and green purchase intentions of consumers in Lesotho", Ethiopian Journal of Environmental Studies and Management, vol. 7, n. 4, pp. 361-370.

KIM H.S. (2006), "Using hedonic and utilitarian shopping motivations to profile inner city consumers", Journal of Shopping Center Research, vol. 13, n. 1, pp. 57-79.

KIM J.H., LENNON S. (2012), "Music and amount of information: do they matter in an online apparel setting?", The International Review of Retail, Distribution and Consumer Research, vol. 22, n. 1, pp. 55-82.

KIM Y.K. (2002), "Consumer value: an application to mall and Internet shopping", International Journal of Retail \& Distribution Management, vol. 30, n. 12, pp. 595-602.

KIM S., LIN J. (2001), “Consumers' perceived importance of and satisfaction with Internet shopping", Electronic Markets, vol. 1, n. 3, pp. 148-154.

KIM S., STOEL L. (2004), "Apparel retailers: websitequality dimensions and satisfaction", Journal of Retailing and Consumer Services, vol. 11, n. 2, pp.
Marco Cioppi

Elisabetta Savelli

Lederica Tombari

atmosphere

tra i visitatori del centro

commerciale 
sinergie Vol. 34, N. 100, 2016

109-117.

KIRTIŞ A.K., KARAHAN F. (2011), “To be or not to be in Social Media arena as the most cost-efficient marketing strategy after the global recession", Procedia-Social and Behavioral Sciences, vol. 24, pp. 260-268.

KOO D.M., JU S.H. (2010), “The interactional effects of atmospherics and perceptual curiosity on emotions and online shopping intention", Computers in Human Behavior, vol. 26, n. 3, pp. 377-388.

KWON D., KNOWN Y.J., SONG Y.-T., LEE R. (2014), “An empirical study on the relationship between user characteristics and quality factors for effective shopping mall websites implementation", in LEE R. (ed.), Software Engineering Research, Management and Applications, Springer International Publishing, pp. 117-127.

ISTAT (2014), "Le tecnologie dell'informazione e della comunicazione nelle imprese", Documento disponibile online: http://www.istat.it/it/ archivio/143752 (ultimo accesso: 7 marzo 2016).

LEE Y., KOZAR K.A. (2006), "Investigating the effect of websitequality on e-business success: an analytic hierarchy process (AHP) approach", Decision Support Systems, vol. 42, n. 3, pp. 1383-1401.

LIANG T.P., LAI H.J. (2002), "Effect of store design on consumer purchases: van empirical study of online bookstores", Information Management, vol. 39, n. 6, pp. 431-44.

LIN J.C.C., LU H. (2000), “Towards an understanding of the behavioral intention to use a web site", International Journal of Information Management, n. 20, pp. 197-208.

LOIACONO E.T., RICHARD T.W., GOODHUE D.L. (2007), "WebQual: an instrument for consumer evaluation of web sites", International Journal of Electronic Commerce, vol. 11, n. 3, pp. 51-87.

MANGANARI E.E., SIOMKOS G.J., VRECHOPOULOS A.P. (2009), "Store atmosphere in web retailing", European Journal of Marketing, vol. 43, n. 9/10, pp. 1140-1153.

MARTIN C.A., TURLEY, L.W. (2004), "Malls and consumption motivation: an exploratory examination of older Generation Y consumers", International Journal of Retail \& Distribution Management, vol. 32, n. 10, pp. 464-475.

MASSICOTTE M., MICHON R., CHEBAT J., SIRGY M., BORGES A. (2011), "Effects of Mall Atmosphere on Mall Evaluation: Teenage Versus Adult Shoppers”, Journal of Retailing and Consumer Services, n. 18, pp. 74-80.

MATTHEWS M., TAYLOR M., SMITH P.B., LIMB M. (2000), “The Unacceptable Flaneur: The Shopping Mall as a Teenage Hangout”, Chilhood 2000, n. 7, pp. 279.

MIRANDA F.J., BENAGIL T.M. (2004), "Quantitative evaluation of commercial websites: An empirica study of Spanish firms", International Journal of Information Management, vol. 24, n. 4, pp. 313-328.

MOLLA-DESCALS A., FRASQUET M., RUIZ-MOLINA M.E., NAVARROSANCHEZ E. (2014), "Determinants of websitetraffic: the case of European fashion apparel retailers", The International Review of Retail, Distribution and Consumer Research, vol. 24, n. 4, pp. 411-430.

NIELSEN J. (2000), Designing Web Usability, New Riders Publishing, Indianapolis.

NIKOLAEVA R. (2006), "E-commerce adoption in the retail sector: empirical insights", International Journal of Retail and Distribution Management, vol. 
34, n. 4/5, pp. 369-387.

NOUR M.A., FADLALLA A. (2000), "A framework for web marketing strategies", Federica Tombari Information Systems Management, vol. 17, n. 2, pp. 41-50.

La percezione della web atmosphere

RANGANATHAN C., GANAPATHY S. (2002), "Key dimensions of business-to- commerciale centro consumer web sites", Information and Management, vol. 39, n. 6, pp. 457-465.

RAPPORTO CENSIS/UCSI (2013), Documento disponibile online: http://www. censis.it/7?shadow_comunicato_stampa=120930 (ultimo accesso: 7 agosto 2015).

REDA S. (1997), "Improved search function seen as a key to Internet shopping", Stores, vol. 79, n. 8, pp. 60-67.

RIEGNER C. (2007), "Word of mouth on the web: the impact of Web 2.0 on consumer purchase decisions", Journal of Advertising Research, vol. 47, n. 4, pp. 436-447.

ROBBINS S., STYLIANOU A. (2003), "Global corporate websites: An empirical investigation of content and design", Information and Management, n. 40, pp. 205-212.

SANDS S., OPPEWAL H., BEVERLAND M. (2008), "The Influence of In-store Experiential Events on Shopping Value Perceptions and Shopping Behavior", Advances in Consumer Research, n. 35, pp. 298-303.

SARI A.A., KUSUMA H.E., TEDJO B. (2015), "A Strategic Planning For A College Student-Segment Shopping Mall”, International Research Journal of Business Studies, vol. 4, n. 2.

SCHAUPP L.C., BELANGER F. (2005), "A conjoint analysis of online consumer satisfaction", Journal of Electronic Commerce Research, vol. 6, n. 2, pp. 95-111.

SHIM S.I., LEE Y. (2011), "Consumer's perceived risk reduction by 3D virtual model”, International Journal of Retail and Distribution Management, vol. 39, n. 12, pp. 945-959.

SHIM B.S., SUH Y.M. (2010), "CRM Strategies for a Small-Sized Online Shopping Mall Based on Association Rules and Sequential Patterns", in: 14th Pacific Asia Conference on Information Systems, PACIS, pp. 355-366.

SCHNEIDERMAN B. (1988), Designing the User Interface: Strategies for Effective Human-Computer Interaction, 3rd edition, Addison Wesley, Reading.

TAN G.W., WEI K.K. (2006), "An empirical study of web browsing: Towards an effective websitedesign ", Electronic Commerce Research and Applications, $\mathrm{n}$. 5, pp. 261-271.

TAYLOR M.J., ENGLAND D. (2006), "Internet marketing: web site navigational design issues", Marketing Intelligence and Planning, vol. 24, n. 1, pp. 77-85.

TORDJMAN A. (1994), "European retailing: convergences, differences and perspectives", International Journal of Retail and Distribution Management, vol. 22, n. 5, pp. 3-19.

TURLEY L.W., MILLIMAN R.E. (2000), "Atmospheric Effects on Shopping Behaviour: a Review of the Experimental Evidence", Journal of Business Research, vol. 49, n. 2, pp. 193-211.

WAN A.H. (2000), "Opportunities to enhance a commercial website", Information and Management, vol. 38, n. 1, pp. 15-21.

WANG L.C., BAKER J., WAGNER J.A., WAKEFIELD K. (2007), "Can a retail web site be social?", Journal of Marketing, vol. 71, n. 3, pp. 143-157. 
sinergie Vol. 34, N. 100, 2016

WANG X., YU C., WEI Y. (2012), “Social media peer communication and impacts on purchase intentions: A consumer socialization framework", Journal of Interactive Marketing, vol. 26, n. 4, pp. 198-208.

WEINBERG D.B. (2000), "Don't keep your internet customers waiting too long at the (virtual) front door", Journal of Interactive Marketing, vol. 14, n. 1, pp. 30-39.

WESTBROOK R.A., BLACK W.C. (1985), “A Motivation-based Shopper Typology”, Journal of Retailing, vol. 61, n. 1, pp. 78-104.

ZVIRAN M., GLEZER C., AVIN I. (2006), "User satisfaction from commercial web sites: the effect of design and use", Information and Management, vol. 43, n. 2, pp. 157-78.

\section{Academic or professional position and contacts}

\section{Marco Cioppi}

Associate Professor of Management

University of Urbino Carlo Bo - Italy

e-mail: marco.cioppi@uniurb.it

\section{Elisabetta Savelli}

Researcher of Management

University of Urbino Carlo Bo - Italy

e-mail: elisabetta.savelli@uniurb.it

\section{Federica Tombari}

Data Mining Consultant - Pesaro - Italy

e-mail: federicatombari@yahoo.it

sinergie italian journal of management

ISSN 0393-5108 DOI $10.7433 / \mathrm{s} 100.2016 .10$ pp. $155-180$

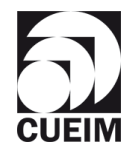

